\title{
Oxy-RVAD support for Lung Transplant in the Absence of Inferior Vena Cava
}

\author{
Kyla Joubert ${ }^{1}$, Takashi Harano ${ }^{2}$, Joseph Pilewski ${ }^{1}$, and Pablo Sanchez ${ }^{1}$ \\ ${ }^{1}$ University of Pittsburgh Medical Center Health System \\ ${ }^{2}$ University of Pittsburgh Medical Center
}

July 24, 2020

\begin{abstract}
Cardiopulmonary bypass and extracorporeal membrane oxygenation are commonly used adjuncts to lung transplantation. These techniques are not without associated morbidity and mortality, and the surgeon must be aware of the possibility of aberrant anatomy that could lead to vascular injury during cannulation. In this report, we describe a patient with congenital absence of the inferior vena cava undergoing lung transplantation who required perioperative cardiopulmonary support. A percutaneous dual lumen cannula, Protek Duo, was connected in an Oxy-RVAD configuration to provide right ventricular and oxygenation support both intraoperatively and postoperatively to this patient.
\end{abstract}

\section{Introduction}

Methods used for cardiac and respiratory support during lung transplantation include cardiopulmonary bypass (CPB), veno-arterial extracorporeal membrane oxygenation (VA-ECMO), and veno-venous extracorporeal membrane oxygenation (VV-ECMO). ${ }^{1}$ These strategies typically require venous drainage by placing a cannula within the inferior vena cava (IVC). However, in rare instances the patient may have anomalies of the IVC, which would preclude this. Absence of the IVC has an incidence of $0.0005 \%$ to $1 \%$ in the general population. ${ }^{2}$ We present a case of a patient with absence of the IVC who required cardiopulmonary support during lung transplantation and the method employed to provide such support.

\section{Case Report}

A 50-year-old-male with cystic fibrosis and multidrug resistant pseudomonas, Achromobacter, methicillin resistant Staph aureus, and recurrent sinusitis was referred for a double lung transplant and concomitant liver transplant. He was declined for a liver transplant due to congenital absence of the IVC. We decided to proceed with double lung transplant only since the synthetic function of the liver was preserved. Other comorbidities included diabetes, compensated cirrhosis, and HIV. He required $4 \mathrm{~L}$ of $\mathrm{O}_{2}$ at rest and $5 \mathrm{~L}$ with exertion, had a mean PA pressure of $21 \mathrm{mmHg}$, an ejection fraction of $65 \%$, and no significant coronary artery disease. MRI and CT showed an absence of normal infrahepatic IVC with enlarged lumbar retro peritoneal collaterals continuing into the azygous. There was normal portal venous flow and hepatic venous flow into the suprahepatic IVC (figure 1). A suitable donor became available. We thought that it would not have been prudent to initiate emergent $\mathrm{CPB}$ or ECMO intraoperatively given his anatomy; therefore, the decision was made to insert the Protek duo prior to the transplantation. For right ventricular and oxygenation support, a 31-Fr Protek Duo cannula was inserted percutaneously into the right internal jugular vein using fluoroscopy and transesophageal echocardiography. The cannula was connected to a modified cardiopulmonary bypass (CPB) machine, in an Oxy-RVAD configuration. The right ventricle (RV) remained decompressed and the patient was well oxygenated throughout the operation. Bilateral sequential lung transplantation required extensive adhesiolysis. The patient had previously undergone several chest tube placements bilaterally and 
doxycycline pleurodesis due to pneumothoraces. The lungs were adherent to the chest well, diaphragm, and pericardium. Right and left lung total ischemic times were 387 and 488 minutes. The Protek duo was left in place for perioperative oxygenation support. The patient remained on Oxy-RAVD support after completion of the case due to a hypokinetic RV and large amount of blood product transfusions. Postoperatively, the CPB machine was converted to a CentriMag System with an inline Quadrox oxygenator. Due to the extensive adhesiolysis the chest was left open and packed. After $48 \mathrm{hrs}$ the patient returned to the OR for chest washout, closure and tracheostomy (figure 2). The patient was decannulated bedside from support on postoperative day 11. Initially the patient was doing very well, however within 3 weeks post lung transplant he developed recurrent Achromobacter sepsis, renal failure requiring dialysis, and shock leading to multisystem organ failure. Due to multiple positive bacterial cultures during broad spectrum antibiotic coverage, the patient received phage therapy. However, after careful consideration with the patient, family, palliative care, and medical ethics, the patient was transitioned to comfort measures only at his request. He expired 7 weeks after transplantation.

\section{Comment}

We present an unusual situation in which a patient requiring lung transplantation had congenital absence of the IVC. In this case the Protek Duo cannula not only allowed for intraoperative support but also postoperative oxygenation.

Aberrant IVC anatomy may lead to significant morbidity and mortality secondary to vascular injuries during cannulation. Wolfhard et al. described a case in which absence of the IVC was discovered during routine coronary bypass grafting. Inspection of the right atrium revealed hepatic vein connection without an IVC. For cardiopulmonary bypass, the SVC and right atrium were cannulated separately and then Y-connected. Post operative imaging revealed bilateral IVC with azygous continuation. ${ }^{3}$ The prevalence of IVC duplication is $0.2 \%-3 \%$. Typically, the IVC ends at the left renal vein, which then crosses anterior to the aorta and joins the right IVC. ${ }^{4}$

$\mathrm{Ng}$ et al. presented a vascular injury in a patient with a double IVC due to ECMO cannula placement. In this case, the left femoral vein was accessed using ultrasound guidance and on routine abdominal x-ray, there was abnormal cannula positioning. A subsequent CT scan revealed a double IVC with venous perforation at the junction of the left IVC and left renal vein. The venous injury was repaired surgically and the cannula position was moved to the right femoral vein which had a normal course. Adequate oxygenation and flow was achieved. ${ }^{5}$

This double lumen cannulation technique as a method of cardiopulmonary bypass has previously been used in the setting of lung transplantation and has demonstrated several advantages. (1) It can be left in place postoperatively for ongoing RV support, (2) avoids complete diversion of pulmonary blood flow therefore reducing allograft ischemia, (3) may reduce the need for arterial access, (4) and allows for bedside decannulation. Caution should be taken in the setting pulmonic valve insufficiency due to possible RV distention. 6

In summary, in a patient with an anatomic variant or absence of the IVC undergoing lung transplantation, satisfactory cardiopulmonary support can be achieved. With the Protek Duo connected to a modified CPB system in an Oxy-RVAD configuration, RV support and adequate oxygenation can be maintained both intra operatively and post operatively. This technique is a relatively simple solution to an otherwise challenging situation.

\section{References:}

1. Magouliotis DE, Tasiopoulou VS, Svokos AA. Extracorporeal membrane oxygenation versus cardiopulmonary bypass during lung transplantation : a meta-analysis. Gen Thorac Cardiovasc Surg . 2018;66(1):3847.

2. Sneed D, Hamdallah I, Sardi A. Absence of the Retrohepatic Inferior Vena Cava: What the Surgeon Should Know. Am Surg . 2005;71(6):502-504. 
3. Wolfhard U, Splittgerber FH, Gocke P, et al. Bilateral inferior vena cava with azygos continuation but without congenital heart disease complicates routine venous cannulation for cardiopulmonary bypass in an adult. Thorac Cardiovasc Surg .1997;45(1):40-42.

4. Huynh PT, Harris JH. Spectrum of Congenital Anomalies of the Inferior Vena Cava: Cross-sectional Imaging Findings. Radiograohics . 2000;20:639-652.

5. Ng PY, Cheuk C, Wong A, et al. Complication of venovenous extracorporeal membrane oxygenation cannulation - the significance of an inferior vena cava anomaly. Clin Case Rep . 2016;4(12):2-4.

6. Fernandez R, Decamp MM, Bharat A. A novel strategy for cardiopulmonary support during lung transplantation. J Thorac Dis . 2018;10(2):142-144.

Figure 1 Contrasted computed tomography of the chest and upper abdomen highlighting with typical lung findings seen in cystic fibrosis along with lack of an intrahepatic IVC.

Figure 2 Chest roentgenograms with Protek Duo inserted into the right internal jugular vein and terminating in the main pulmonary artery with (A) open chest, (B) closed chest and tracheostomy, and (C) finally, with the Protek Duo removed.

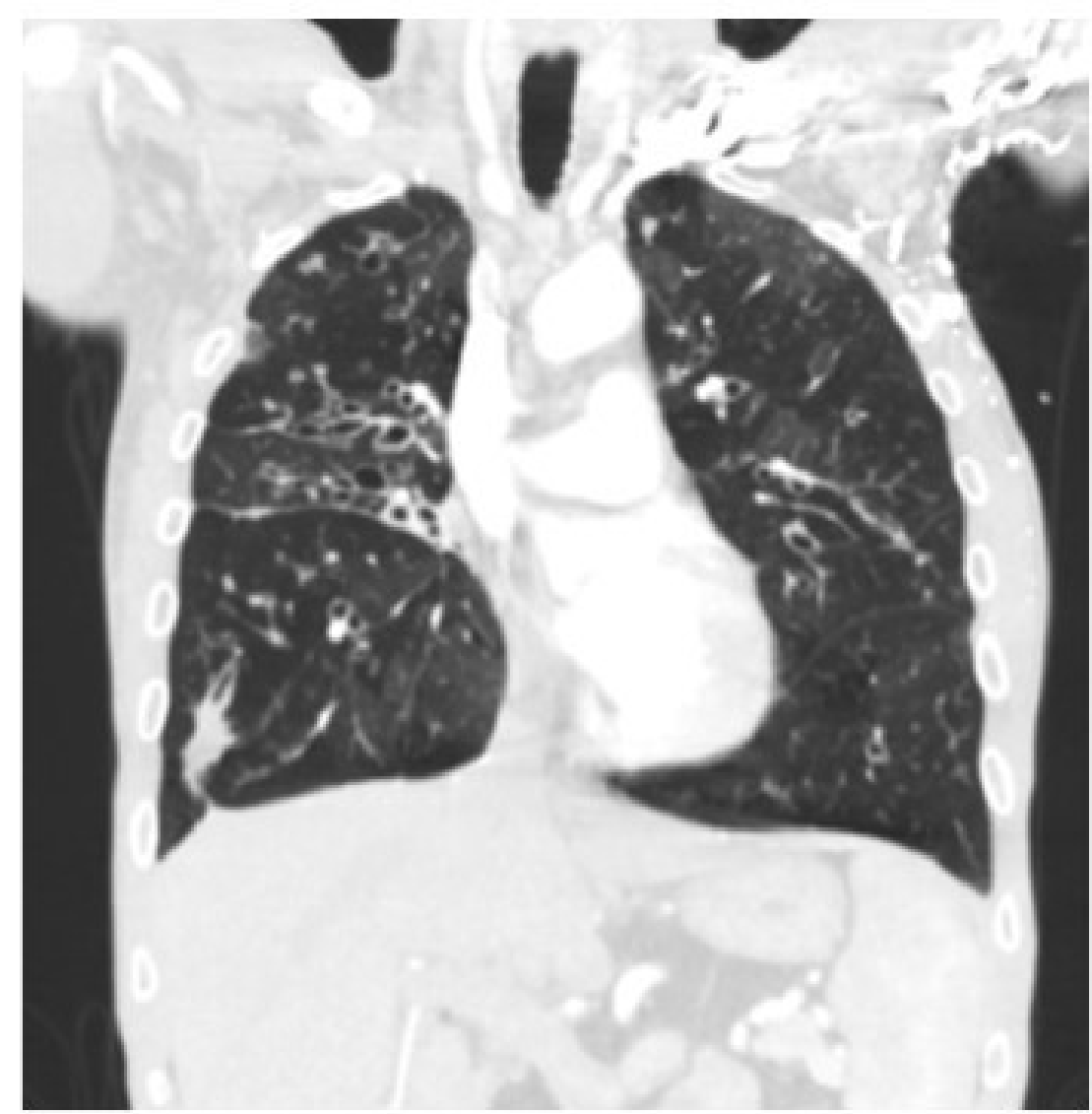




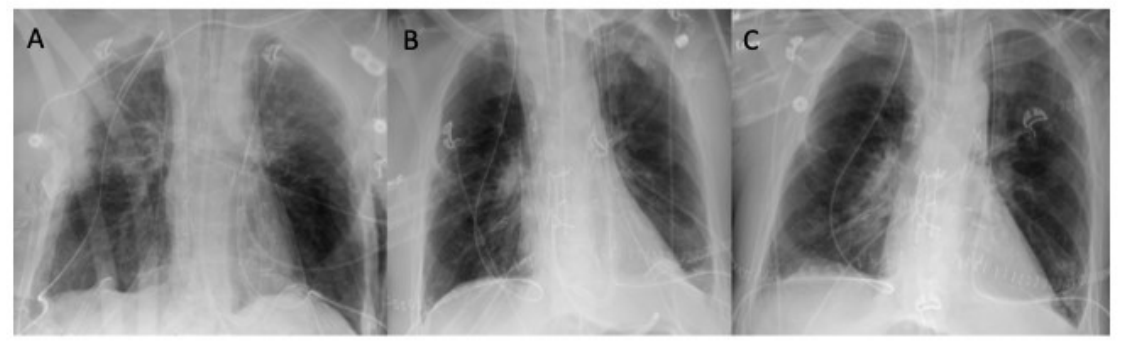

